\title{
Quantum Complexities of Ordered Searching, Sorting, and Element Distinctness ${ }^{\star}$
}

\author{
Peter Høyer ${ }^{1, \star \star}$, Jan Neerbek ${ }^{2}$, and Yaoyun $\mathrm{Shi}^{3}$ \\ 1 Dept. of Comp. Sci., University of Calgary, Alberta, Canada T2N 1N4 \\ hoyer@cpsc.ucalgary.ca \\ 2 Dept. of Comp. Sci., University of Aarhus, DK-8000 Århus C, Denmark \\ neerbek@daimi.au.dk \\ 3 Dept. of Comp. Sci., Princeton University, Princeton, NJ 08544, USA \\ shiyy@cs.princeton.edu
}

\begin{abstract}
We consider the quantum complexities of the following three problems: searching an ordered list, sorting an un-ordered list, and deciding whether the numbers in a list are all distinct. Letting $N$ be the number of elements in the input list, we prove a lower bound of $\frac{1}{\pi}(\ln (N)-1)$ accesses to the list elements for ordered searching, a lower bound of $\Omega(N \log N)$ binary comparisons for sorting, and a lower bound of $\Omega(\sqrt{N} \log N)$ binary comparisons for element distinctness. The previously best known lower bounds are $\frac{1}{12} \log _{2}(N)-O(1)$ due to Ambainis, $\Omega(N)$, and $\Omega(\sqrt{N})$, respectively. Our proofs are based on a weighted all-pairs inner product argument.

In addition to our lower bound results, we give a quantum algorithm for ordered searching using roughly $0.631 \log _{2}(N)$ oracle accesses. Our algorithm uses a quantum routine for traversing through a binary search tree faster than classically, and it is of a nature very different from a faster algorithm due to Farhi, Goldstone, Gutmann, and Sipser.
\end{abstract}

\section{Introduction}

The speedups of quantum algorithms over classical algorithms have been a main reason for the current interests on quantum computing. One central question regarding the power of quantum computing is: How much speedup is possible? Although dramatic speedups seem possible, as in the case of Shor's 16 algorithms for factoring and for finding discrete logarithms, provable speedups are found only in restricted models such as the black box model.

In the black box model, the input is given as a black box, so that the only way the algorithm can obtain information about the input is via queries, and the complexity measure is the number of queries. Many problems that allow provable quantum speedups can be formulated in this model, an example being the unordered search problem considered by Grover [14]. Several tight lower

\footnotetext{
* Research supported by the EU fifth framework program QAIP, IST-1999-11234, and the National Science Foundation under grant CCR-9820855.

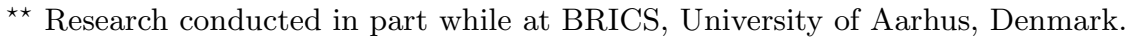


bounds are now known for this model, most of them being based on techniques introduced in [5, 3:,2].

We study the quantum complexities of the following three problems.

Ordered searching Given a list of numbers $x=\left(x_{0}, x_{1}, \ldots, x_{N-1}\right)$ in nondecreasing order and some number $y$, find the minimal $i$ such that $y \leq x_{i}$. We assume that $x_{N-1}=\infty>y$ so that the problem is always well-defined.

Sorting Given a list of numbers $x=\left(x_{0}, x_{1}, \ldots, x_{N-1}\right)$, output a permutation $\sigma$ on the set $\{0, \ldots, N-1\}$ so that the list $\left(x_{\sigma(0)}, x_{\sigma(1)}, \ldots, x_{\sigma(N-1)}\right)$ is in non-decreasing order.

Element distinctness Given a list of numbers $x=\left(x_{0}, x_{1}, \ldots, x_{N-1}\right)$, are they all distinct?

These problems are closely related and are among the most fundamental and most studied problems in the theory of algorithms. They can also be formulated naturally in the black box model. For the ordered searching problem, we consider queries of the type " $x_{i}=$ ?", and for the sorting and element distinctness problems, we consider queries of the type "Is $x_{i}<x_{i^{\prime}}$ ?", which are simply binary comparisons. Let $H_{i}=\sum_{k=1}^{i} \frac{1}{k}$ denote the $i$ th harmonic number. We prove a lower bound for each of these three problems.

Theorem 1. Any quantum algorithm for ordered searching that errs with probability at most $\epsilon \geq 0$ requires at least

$$
(1-2 \sqrt{\epsilon(1-\epsilon)}) \frac{1}{\pi}\left(H_{N}-1\right)
$$

queries to the oracle. In particular, any exact quantum algorithm requires more than $\frac{1}{\pi}(\ln (N)-1) \approx 0.220 \log _{2} N$ queries.

Theorem 2. Any comparison-based quantum algorithm for sorting that errs with probability at most $\epsilon \geq 0$ requires at least

$$
(1-2 \sqrt{\epsilon(1-\epsilon)}) \frac{N}{2 \pi}\left(H_{N}-1\right)
$$

comparisons. In particular, any exact quantum algorithm requires more than $\frac{N}{2 \pi}(\ln (N)-1) \approx 0.110 N \log _{2} N$ comparisons.

Theorem 3. Any comparison-based quantum algorithm for element distinctness that errs with probability at most $\epsilon \geq 0$ requires at least

$$
(1-2 \sqrt{\epsilon(1-\epsilon)}) \frac{\sqrt{N}}{2 \pi}\left(H_{N}-1\right)
$$

comparisons. 
The previously best known quantum lower bound for ordered searching is $\frac{1}{12} \log _{2}(N)-O(1)$, due to Ambainis [1]. For comparison-based sorting and element distinctness, the previously best known quantum lower bounds are respectively $\Omega(N)$ and $\Omega(\sqrt{N})$, both of which can be proven in many ways.

We prove our lower bounds by utilizing what we refer to as a weighted allpairs inner product argument, or a probabilistic adversary argument. This proof technique is based on the work of Bennett, Bernstein, Brassard, and Vazirani [5] and Ambainis [2].

Farhi, Goldstone, Gutmann, and Sipser [12] have given an exact quantum algorithm for ordered searching using roughly $0.526 \log _{2}(N)$ queries. We provide an alternative quantum algorithm that is exact and uses $\log _{3}(N)+O(1) \approx$ $0.631 \log _{2}(N)$ queries. Our construction is radically different from the construction proposed by Farhi et al. [12], and these are the only constructions known leading to quantum algorithms using at most $c \log _{2}(N)$ queries for some constant $c$ strictly less than 1 .

Whereas most quantum algorithms are based on Fourier transforms and amplitude amplification [7], our algorithm is based on binary search trees. We initiate several applications of the binary search algorithm in quantum parallel and let them find the element we are searching for in teamwork. By cooperating, these applications can traverse the binary search tree faster than classically, hereby reducing the complexity from $\log _{2}(N)$ to roughly $\log _{3}(N)$.

There are at least three reasons why the quantum complexities of the three problems are of interest. Firstly because of their significance in algorithmics in general. Secondly because these problems possess some symmetries and periodicities of a different nature than other studied problems in quantum algorithmics. Determining symmetries and periodicities seems to be a primary ability of quantum computers and it is not at all clear how far-reaching this skill is. Thirdly because searching and sorting represent non-Boolean non-symmetric functions. A (partial) function is said to be symmetric if it is invariant under permutation of its input. Only few non-trivial quantum bounds for non-Boolean and non-symmetric functions are known.

The rest of the paper is organized as follows. We first discuss the model in Sect. 2, present our general technique for proving lower bounds in Sect. 3.1, and then apply it to the three problems in Sects. 3.2 3.4. We give our quantum algorithm for ordered searching in Sect. 4 and conclude in Sect. 5 .

\section{Quantum black box computing}

We give a formal definition of the black box model, which is slightly different from, but equivalent to, the definition of Beals, Buhrman, Cleve, Mosca, and de Wolf given in [3]. Fix some positive integer $N>0$. The input $x=$ $\left(x_{0}, \ldots, x_{N-1}\right) \in\{0,1\}^{N}$ is given as an oracle, and the only way we can access 
the bits of the oracle is via queries. A query implements the operator

$$
\mathrm{O}_{x}: \quad|z ; i\rangle \longmapsto\left\{\begin{aligned}
(-1)^{x_{i}}|z ; i\rangle & \text { if } 0 \leq i<N \\
|z ; i\rangle & \text { if } i \geq N
\end{aligned}\right.
$$

Here $i$ and $z$ are non-negative integers. By a query to oracle $x$ we mean an application of the unitary operator $\mathrm{O}_{x}$. We sometimes refer to $\mathrm{O}_{x}$ as the oracle. A quantum algorithm $\mathrm{A}$ that uses $T$ queries to an oracle $\mathrm{O}$ is a unitary operator of the form

$$
\mathrm{A}=(\mathrm{UO})^{T} \mathrm{U}
$$

We always apply algorithm $\mathrm{A}$ on the initial state $|0\rangle$. For every integer $j \geq 0$ and every oracle $x$, let

$$
\left|\psi_{x}^{j}\right\rangle=\left(\mathrm{UO}_{x}\right)^{j} \mathrm{U}|0\rangle
$$

denote the state after $j$ queries, given oracle $x$. After applying $\mathrm{A}$, we always measure the final state in the computational basis.

Consider the computation of some function $f: S \rightarrow\{0,1\}^{m}$, where $S \subseteq$ $\{0,1\}^{N}$. We say that algorithm A computes $f$ with error probability bounded by $\epsilon$, for some constant $\epsilon$ with $0 \leq \epsilon<1 / 2$, if for any $x \in S$, the probability of observing $f(x)$ when the $m$ rightmost bits of $\left|\psi_{x}^{T}\right\rangle$ are measured is at least $1-\epsilon$.

\section{Lower bounds}

\subsection{General technique}

We use the notation of Sect. 2. For any $\epsilon \geq 0$, let $\epsilon^{\prime}=2 \sqrt{\epsilon(1-\epsilon)}$.

The computation always starts in the same initial state $|0\rangle$, so for all oracles $x \in S$ we have $\left|\psi_{x}^{0}\right\rangle=|0\rangle$. If for two input oracles $x, y \in S$, the correct answers are different, i.e., if $f(x) \neq f(y)$, then the corresponding final states $\left|\psi_{x}^{T}\right\rangle$ and $\left|\psi_{y}^{T}\right\rangle$ must be almost orthogonal.

Lemma 4. For all oracles $x, y \in S$ so that $f(x) \neq f(y),\left|\left\langle\psi_{x}^{T} \mid \psi_{y}^{T}\right\rangle\right| \leq \epsilon^{\prime}$.

Now consider a probability distribution over those pairs of inputs $(x, y) \in$ $S \times S$ for which $f(x) \neq f(y)$. For each integer $j \geq 0$, we use the following quantity to quantify the average progress of the algorithm in distinguishing any two inputs after applying $(\mathrm{UO})^{j} \mathrm{U}$,

$$
W_{j}=\mathbf{E}_{(x, y)}\left[\left\langle\psi_{x}^{j} \mid \psi_{y}^{j}\right\rangle\right] .
$$

Observe that $W_{0}=1$ and that $W_{T} \leq \epsilon^{\prime}$ by Lemma 4 . By proving that for every $j$ with $0 \leq j<T$, we have $\left|W_{j}-W_{j+1}\right| \leq \delta$, we conclude that $T \geq\left(1-\epsilon^{\prime}\right) / \delta$. 
For simplicity of presentation, we scale the probabilities by using a weight function $\omega: S \times S \rightarrow \mathbb{R}^{+}$. From now on, we use the following definition of $W_{j}$ to quantify the overall progress of the algorithm,

$$
W_{j}=\sum_{x, y \in S} \omega(x, y)\left\langle\psi_{x}^{j} \mid \psi_{y}^{j}\right\rangle .
$$

Our technique is a natural generalization of Ambainis' approach [2], which uses uniform distributions over subsets of $S \times S$. Our lower bound proofs imply that non-uniform distributions can give better lower bounds. Clearly, finding a "good" distribution is an important step in applying our technique. Another important step is to find a tight bound on the progress after each oracle query.

We end this subsection by introducing some notation and stating two lemmas we require when bounding the progress. For every $i \geq 0$, let $\mathrm{P}_{i}=\sum_{z \geq 0}|z ; i\rangle\langle z ; i|$ denote the projection operator onto the subspace querying the $i$ th oracle bit. For $i<0$, operator $\mathrm{P}_{i}$ is taken as the zero projection. The following lemma, which may be proven by the Cauchy-Schwarz inequality, bounds the quantified progress that one oracle query makes in distinguishing two inputs $x$ and $y$.

Lemma 5. For any oracles $x, y \in\{0,1\}^{N}$, and any integer $j \geq 0$,

$$
\left|\left\langle\psi_{x}^{j} \mid \psi_{y}^{j}\right\rangle-\left\langle\psi_{x}^{j+1} \mid \psi_{y}^{j+1}\right\rangle\right| \leq 2 \sum_{i: x_{i} \neq y_{i}} \| \mathrm{P}_{i}\left|\psi_{x}^{j}\right\rangle\|\cdot\| \mathrm{P}_{i}\left|\psi_{y}^{j}\right\rangle \| .
$$

We sometimes write $\left|\psi_{x}\right\rangle$ as shorthand for $\left|\psi_{x}^{j}\right\rangle$ once integer $j$ is fixed.

Let $A=\left[\alpha_{k, \ell}\right]_{1 \leq k, \ell<\infty}$ be the Hilbert matrix with $\alpha_{k, \ell}=1 /(k+\ell-1)$, and $\|\cdot\|_{2}$ be the spectral norm, i.e., for any complex-valued matrix $M \in \mathbb{C}^{m \times m}$, the norm $\|M\|_{2}$ is defined as $\max \left\{\|M x\|_{2}\right\}$, where the maximum is taken over all unit vectors $x \in \mathbb{C}^{m}$. Let $B_{N}=\left[\beta_{k, \ell}\right]_{1 \leq k, \ell \leq N}$ be the matrix where entry $\beta_{k, \ell}$ is $\frac{1}{k+\ell-1}$ if $k+\ell \leq N+1$, and 0 otherwise. Clearly $\left\|B_{N}\right\|_{2} \leq\|A\|_{2}$ for any $N>0$. Our lower bound proofs rely on the following property of the Hilbert matrix.

Lemma 6 (E.g.: Choi [10]). $\|A\|_{2}=\pi$. Hence, $\left\|B_{N}\right\|_{2} \leq \pi$.

\subsection{Lower bound for ordered searching}

The first non-trivial quantum lower bound on ordered searching proven was $\Omega\left(\sqrt{\log _{2}(N)} / \log _{2} \log _{2}(N)\right)$, due to Buhrman and de Wolf $[9$ by an ingenious reduction from the PARITY problem. Farhi, Goldstone, Gutmann, and Sipser 11$]$ improved this to $\log _{2}(N) / 2 \log _{2} \log _{2}(N)$, and Ambainis 迫 then proved the previously best known lower bound of $\frac{1}{12} \log _{2}(N)-O(1)$. In 11. 1, they use, as we do here, an inner product argument along the lines of [5]. In this section, we improve the lower bound by a constant factor.

For the purpose of proving the lower bound, we assume that each of the $N$ input numbers is either 0 or 1 , and that the input does not consist of all zeroes. That is, the set $S$ of possible inputs are the ordered $N$-bit strings of non-zero Hamming weight. The search function $f: S \rightarrow\{0,1\}^{m}$ is defined by 
$f(x)=\min \left\{0 \leq i<N \mid x_{i}=1\right\}$, where we identify the result $f(x)$ with its binary encoding as a bit-string of length $m=\left\lceil\log _{2}(N)\right\rceil$. As our weight function $\omega$, we choose the inverse of the difference in Hamming weights,

$$
\omega(x, y)= \begin{cases}\frac{1}{f(y)-f(x)} & \text { if } 0 \leq f(x)<f(y)<N \\ 0 & \text { otherwise. }\end{cases}
$$

With this choice, we have that $W_{0}=N H_{N}-N$ and by Lemma 1 also that $W_{T} \leq \epsilon^{\prime} W_{0}$. Theorem 1 then follows from the next lemma.

Lemma 7. For every $j$ with $0 \leq j<T$ we have that $\left|W_{j}-W_{j+1}\right| \leq \pi N$.

Proof. As shorthand, we write $\left|\psi_{f(x)}\right\rangle$ for $\left|\psi_{x}^{j}\right\rangle$. By Lemma 5 ,

$$
\begin{aligned}
\left|W_{j}-W_{j+1}\right| & \leq 2 \sum_{k=0}^{N-2} \sum_{\ell=k+1}^{N-1} \frac{1}{\ell-k} \sum_{i=k}^{\ell-1} \| \mathrm{P}_{i}\left|\psi_{k}\right\rangle\|\cdot\| \mathrm{P}_{i}\left|\psi_{\ell}\right\rangle \| \\
& =2 \sum_{d=1}^{N-1} \sum_{i=0}^{d-1} \frac{1}{d} \sum_{k=0}^{N-d-1} \| \mathrm{P}_{k+i}\left|\psi_{k}\right\rangle\|\cdot\| \mathrm{P}_{k+i}\left|\psi_{k+d}\right\rangle \| .
\end{aligned}
$$

Let vectors $\gamma=\left[\gamma_{i}\right]_{0 \leq i<N-1} \in \mathbb{R}^{N-1}$ and $\delta=\left[\delta_{i}\right]_{0 \leq i<N-1} \in \mathbb{R}^{N-1}$ be defined by

$$
\gamma_{i}=\left(\sum_{k=0}^{N-1} \| \mathrm{P}_{k+i}\left|\psi_{k}\right\rangle \|^{2}\right)^{1 / 2} \quad \text { and } \quad \delta_{i}=\left(\sum_{k=0}^{N-1} \| \mathrm{P}_{k-i-1}\left|\psi_{k}\right\rangle \|^{2}\right)^{1 / 2}
$$

Then, by the Cauchy-Schwarz inequality,

$$
\left|W_{j}-W_{j+1}\right| \leq 2 \sum_{d=1}^{N-1} \sum_{i=0}^{d-1} \frac{1}{d} \gamma_{i} \delta_{d-i-1}=2 \gamma^{t} B_{N} \delta
$$

where $t$ denotes matrix transposition. Since each vector $\left|\psi_{k}\right\rangle$ is of unit norm, we have $\|\gamma\|_{2}^{2}+\|\delta\|_{2}^{2} \leq N$, so $\|\gamma\|_{2}\|\delta\|_{2} \leq N / 2$. The matrix product $2 \gamma^{t} B_{N} \delta$ is upper bounded by $2\|\gamma\|_{2} \cdot\left\|B_{N}\right\|_{2} \cdot\|\delta\|_{2}$, which is at most $\pi N$ by Lemma 6 .

\subsection{Lower bound for sorting}

We assume that the $N$ numbers to be sorted, $x=\left(x_{0}, \ldots, x_{N-1}\right)$, correspond to some permutation $\sigma$ on $\{0,1, \ldots, N-1\}$. That is, $x_{i}=\sigma(i)$ for every $0 \leq i<$ $N$. We assume the input to the quantum algorithm is the comparison matrix $M_{\sigma}=\left[m_{i i^{\prime}}\right]_{0 \leq i, i^{\prime}<N}$ with

$$
m_{i i^{\prime}}= \begin{cases}1 & \text { if } \sigma(i)<\sigma\left(i^{\prime}\right) \\ 0 & \text { otherwise }\end{cases}
$$


One comparison corresponds to one application of the oracle operator

$$
\mathrm{O}_{\sigma}=\sum_{z \geq 0} \sum_{i, i^{\prime} \geq 0}(-1)^{m_{i i^{\prime}}}\left|z ; i, i^{\prime}\right\rangle\left\langle z ; i, i^{\prime}\right| .
$$

To simplify notation, we sometimes identify the input $M_{\sigma}$ with the underlining permutation $\sigma$.

For every pair $\left\{i, i^{\prime}\right\}$ of indices with $0 \leq i, i^{\prime}<N$, let

$$
\mathrm{P}_{i i^{\prime}}=\sum_{z \geq 0}\left|z ; i, i^{\prime}\right\rangle\left\langle z ; i, i^{\prime}\left|+\sum_{z \geq 0}\right| z ; i^{\prime}, i\right\rangle\left\langle z ; i^{\prime}, i\right|
$$

denote the projection operator onto the subspace comparing the $i$ th and $\left(i^{\prime}\right)$ th elements. For any vector $|\psi\rangle$, we use $\left|\psi \uparrow_{\sigma, k, \ell}\right\rangle$ as shorthand for $\mathrm{P}_{\sigma^{-1}(k), \sigma^{-1}(\ell)}|\psi\rangle$.

For every permutation $\sigma$, and every integers $0 \leq k \leq N-2$ and $1 \leq d \leq$ $N-1-k$, define a new permutation,

$$
\sigma^{(k, d)}=(k, k+1, \ldots, k+d) \circ \sigma .
$$

If $\tau=\sigma^{(k, d)}$, then

$$
\sigma^{-1}(i)= \begin{cases}\tau^{-1}(k) & \text { if } i=k+d \\ \tau^{-1}(i+1) & \text { if } k \leq i<k+d \\ \tau^{-1}(i) & \text { otherwise }\end{cases}
$$

This implies that the comparison matrices $M_{\sigma}$ and $M_{\tau}$ differ only on the following pairs of entries,

$$
\left\{\sigma^{-1}(k+d), \sigma^{-1}(k+i)\right\}=\left\{\tau^{-1}(k), \tau^{-1}(k+i+1)\right\}
$$

for all $i$ with $0 \leq i<d$.

Informally, if $M_{\sigma}$ corresponds to some list $x$, then $M_{\tau}$ corresponds to the list $y$ obtained by replacing the element of rank $k+d$ in $x$ by a new element of rank $k$ (the element in $x$ that had rank $k$ then has rank $k+1$ in $y$, etc.). The only way the algorithm can distinguish $\sigma$ from $\tau$ is by comparing the element of rank $k+d$ in $x$ with one of the $d$ elements of rank $k+i$ for some $0 \leq i<d$.

We choose the following weight function,

$$
\omega(\sigma, \tau)= \begin{cases}\frac{1}{d} & \text { if } \tau=\sigma^{(k, d)} \text { for some } k \text { and } d \\ 0 & \text { otherwise. }\end{cases}
$$

Then one may verify that $W_{0}=N !\left(N H_{N}-N\right)$, and $W_{T} \leq \epsilon^{\prime} W_{0}$. To prove Theorem 2, we need only to prove the following lemma.

Lemma 8. For any $j$ with $0 \leq j<T,\left|W_{j}-W_{j+1}\right| \leq 2 \pi N$ !. 
Proof. Similar to the proof of Lemma ㄱ. By Lemma 5 and (13),

$$
\left|W_{j}-W_{j+1}\right| \leq 2 \sum_{d=1}^{N-1} \sum_{i=0}^{d-1} \frac{1}{d} \sum_{\sigma} \sum_{k=0}^{N-d-1}\left\|\left|\psi_{\sigma}\left\lceil_{\sigma, k+d, k+i}\right\rangle\|\cdot\|\right| \psi_{\sigma^{(k, d)}} \uparrow_{\sigma, k+d, k+i}\right\rangle \| .
$$

Let $\gamma=\left[\gamma_{i}\right]_{1 \leq i<N} \in \mathbb{R}^{N-1}$ be such that $\gamma_{i}=\left(\sum_{\sigma} \sum_{\ell=0}^{N-1}\left\|\mid \psi_{\sigma}\left\lceil_{\sigma, \ell, \ell+i}\right\rangle\right\|^{2}\right)^{1 / 2}$, where we let $\ell$ range from 0 to $N-1$ and simply set the thus caused undefined projection operators to be zero operators. Then by (12),

$$
\sum_{\sigma} \sum_{k=0}^{N-d-1} \|\left|\psi_{\sigma^{(k, d)}} \uparrow_{\sigma, k+d, k+i}\right\rangle\left\|^{2}=\sum_{\tau} \sum_{k=0}^{N-d-1}\right\|\left|\psi_{\tau} \uparrow_{\tau, k, k+i+1}\right\rangle \|^{2} \leq \gamma_{i+1}^{2} .
$$

Applying the Cauchy-Schwarz inequality, and in analogy with (10),

$$
\left|W_{j}-W_{j+1}\right| \leq 2 \sum_{d=1}^{N-1} \sum_{i=0}^{d-1} \frac{1}{d} \gamma_{d-i} \gamma_{i+1}=2 \gamma^{t} B_{N-1} \gamma
$$

Since $\|\gamma\|_{2}^{2} \leq N$ !, we conclude that $\left|W_{j}-W_{j+1}\right| \leq 2 \pi N$ !.

\subsection{Lower bound for element distinctness}

We modify the adversary for sorting as follows. As in Sect. 3.3, when we talk about permutations, the underlying set is $\{0,1, \ldots, N-1\}$.

Definition 9. An annotated permutation is a permutation $\tau$ with a marker on a single element $r_{\tau}$ for some $0 \leq r_{\tau}<N-1$.

For every permutation $\sigma$, and every integers $k$ and $d$ as in Sect. 3.3, the annotated permutation $\tau=\sigma^{(k, d)}$ is the same permutation as in (11) but with the rank $k$ element marked. The only places where $M_{\sigma}$ and $M_{\tau}$ differ, are at the same entries as those in (13).

We use the same weight function as in (14). Then $W_{0}=N !\left(N H_{N}-N\right)$ and $W_{T} \leq \epsilon^{\prime} W_{0}$. We need only to prove the following lemma.

Lemma 10. For any integer $j$ with $0 \leq j<T,\left|W_{j}-W_{j+1}\right| \leq 2 \pi N ! \sqrt{N}$.

Proof. Almost identical to the proof for Lemma 8, except that we now require a second vector $\delta=\left[\delta_{i}\right]_{1 \leq i<N} \in \mathbb{R}^{N-1}$ with $\left.\delta_{i}=\left(\sum_{\tau} \|\left|\psi_{\tau}\right|_{\tau, r_{\tau}, r_{\tau}+i}\right\rangle \|^{2}\right)^{1 / 2}$. Then by (12),

$$
\sum_{\sigma} \sum_{k=0}^{N-d-1} \|\left|\psi_{\sigma^{(k, d)}} \uparrow_{\sigma, k+d, k+i}\right\rangle\left\|^{2}=\sum_{\tau: r_{\tau}<N-d}\right\|\left|\psi_{\tau} \uparrow_{\tau, r_{\tau}, r_{\tau}+i+1}\right\rangle \|^{2} \leq \delta_{i+1}^{2} .
$$


In analogy with (15), we have

$$
\left|W_{j}-W_{j+1}\right| \leq 2 \sum_{d=1}^{N-1} \sum_{i=0}^{d-1} \frac{1}{d} \gamma_{d-i} \delta_{i+1}=2 \gamma^{t} B_{N-1} \delta .
$$

Besides having $\|\gamma\|^{2} \leq N$ ! as in the proof of Lemma 8, we also have that

$$
\|\delta\|^{2}=\sum_{i=1}^{N-1} \sum_{\tau} \|\left|\psi_{\tau} \uparrow_{\tau, r_{\tau}, r_{\tau}+i}\right\rangle \|^{2} \leq N !(N-1) \leq N ! N .
$$

Therefore, $\left|W_{j}-W_{j+1}\right| \leq 2 \pi \sqrt{N !} \sqrt{N ! N}=2 \pi N ! \sqrt{N}$.

\section{$4 \quad \mathrm{~A}_{\log _{3}}(N)$ algorithm for ordered searching}

We begin by considering binary search trees on which our quantum algorithm is based. Let $\mathcal{T}$ be a binary tree with $N \geq 2$ leaves. We put colored pebbles on the (internal) vertices of $\mathcal{T}$ subject to the following 2 conditions:

(A) on every path from the root of $\mathcal{T}$ to a leaf, there is exactly 1 pebble of each color, and

(B) the number of pebbles $p_{v}$ on any vertex $v \in \mathcal{T}$ is at least as large as the total number of pebbles on its proper ancestors.

We say that $\mathcal{T}$ is covered by $N^{\prime}$ pebbles if we can satisfy the 2 above conditions using at most $N^{\prime}$ pebbles of each color. We want to minimize the maximum number $N^{\prime}$ of pebbles used of any color. We say a covering is fair if it uses the same number of pebbles of every color. We say a covering is tight if, for all vertices $v \in \mathcal{T}$, we have that $p_{v}$ equals the total number of pebbles on its proper ancestors, or there are no pebbles on any of the ancestors of $v$. We require the following two lemmas.

Lemma 11. For every even integer $N \geq 2$, there exists a binary tree with $N$ leaves that can be fairly and tightly covered by $N^{\prime}=\left\lfloor\frac{1}{3} N+\log _{2}(N)\right\rfloor$ pebbles using $2^{s}$ colors, where $s=\left\lfloor\log _{4}(N / 2)\right\rfloor$.

Lemma 12. Let integer-valued function $\tilde{F}$ be recursively defined by

$$
\tilde{F}(N)= \begin{cases}\tilde{F}\left(\left\lfloor\frac{1}{3} N+\log _{2}(N)+1\right\rfloor\right)+1 & \text { if } N>8 \\ 1 & \text { if } N \leq 8 .\end{cases}
$$

Then $\tilde{F}(N)=\log _{3}(N)+O(1)$.

As in Sect. 3.2, we assume the oracle $x=\left(x_{0}, \ldots, x_{N-1}\right) \in\{0,1\}^{N}$ is a binary string of non-zero Hamming weight. The problem is to determine the leftmost 1 in $x$, that is, to compute $f(x)=\min \left\{0 \leq i<N \mid x_{i}=1\right\}$. Let $\mathcal{T}$ 
be a binary tree with $N$ leaves for which Lemma 11 holds. Let $s=\left\lfloor\log _{4}(N / 2)\right\rfloor$ and $N^{\prime}=\left\lfloor\frac{1}{3} N+\log _{2}(N)\right\rfloor$ be as in the lemma. We label the $N$ leaves of $\mathcal{T}$ by $\{0, \ldots, N-1\}$ from left to right. Let $\ell_{f(x)}$ denote the leaf labelled by $f(x)$, and let $\mathcal{P}$ denote the path from the root of $\mathcal{T}$ to the parent of $\ell_{f(x)}$. We think of $\mathcal{P}$ as the path the classical search algorithm would traverse if searching for $f(x)$ in tree $\mathcal{T}$.

Let $\mathcal{C}=\left\{c_{0}, \ldots, c_{2_{-1}}\right\}$ be the set of $2^{s}$ colors used in Lemma 11. For each color $c \in \mathcal{C}$, let $V_{c}$ denote the set of vertices in $\mathcal{T}$ populated by a pebble of color $c$. By Condition (A), there are at most $N^{\prime}$ such vertices, that is, $\left|V_{c}\right| \leq N^{\prime}$. Let $v_{c}$ denote the unique vertex in $V_{c}$ that is on path $\mathcal{P}$. We think of vertex $v_{c}$ as the root of the subtree "containing" leaf $\ell_{f(x)}$. Note that, by definition, $v_{c} \in \mathcal{P}$ for every color $c \in \mathcal{C}$, and that $\sum_{v \in \mathcal{P}} p_{v}=2^{s}$ by Condition $(\mathrm{A})$.

Our algorithm utilizes 3 unitary operators, $\mathrm{U}_{1}, \mathrm{O}_{x}^{\prime}$, and $\mathrm{U}_{2}$. The first operator, $\mathrm{U}_{1}$, is defined by

$$
\mathrm{U}_{1}: \quad|v\rangle|0\rangle \longmapsto|v\rangle\left(\frac{1}{\sqrt{p_{v}}} \sum_{c}|c\rangle\right) \quad(v \in \mathcal{T})
$$

where the summation is over all colors $c \in \mathcal{C}$ that are represented by a pebble on vertex $v$. We refer to $\mathrm{U}_{1}$ as the coloring operator and its inverse as the un-coloring operator.

The query operator $\mathrm{O}_{x}^{\prime}$ is defined by

$$
\mathrm{O}_{x}^{\prime}:|v\rangle \longmapsto\left\{\begin{aligned}
\left|v ; x_{i}\right\rangle & \text { if there are no pebbles on the parent of } v \\
(-1)^{x_{i}}|v\rangle & \text { otherwise, }
\end{aligned}\right.
$$

where $i$ denotes the label of the rightmost leaf in the left subtree of vertex $v$. Query operator $\mathrm{O}_{x}^{\prime}$ is clearly unitary (or rather, can be extended to a unitary operator since it is only defined on a proper subspace). Operator $\mathrm{O}_{x}^{\prime}$ is slightly different from, but equivalent to, the query operator defined in Sect. 2. It mimics the classical search algorithm by querying the bit $x_{i}$ that corresponds to the rightmost leaf in the left subtree of $v$.

We also use a unitary operator $U_{2}$ that maps each vertex to a superposition over the leaves in its subtree. For every vertex and leaf $u$ in $\mathcal{T}$, let $\mathcal{L}(u)$ denote the set of leaves in the subtree rooted at $u$, and let

$$
\left|\Phi_{u}\right\rangle=\sum_{\ell \in \mathcal{L}(u)} \frac{1}{\sqrt{2^{d(u, \ell)}}}|\ell\rangle
$$

where $d(u, \ell)$ denotes the absolute value of the difference in depths of $u$ and leaf $\ell$. The unitary operator $U_{2}$ is (partially) defined as follows. For every vertex $v \in \mathcal{T}$ with no pebbles on its parent,

$$
\begin{aligned}
& |v ; 0\rangle \longmapsto\left|\Phi_{\operatorname{right}(v)}\right\rangle \\
& |v ; 1\rangle \longmapsto\left|\Phi_{\operatorname{left}(v)}\right\rangle,
\end{aligned}
$$


and for every vertex $v \in \mathcal{T}$ with pebbles on its parent,

$$
|v\rangle \longmapsto \frac{1}{\sqrt{2}}\left(\left|\Phi_{\operatorname{right}(v)}\right\rangle-\left|\Phi_{\operatorname{left}(v)}\right\rangle\right) .
$$

Here left $(v)$ denotes the left child of $v$, and right $(v)$ the right child.

Our quantum algorithm starts in the initial state $|0\rangle$ and produces the final state $\left|\ell_{f(x)}\right\rangle$. Let $F(N)$ denote the number of queries used by the algorithm on an oracle $x$ of size $N$.

1. We first set up a superposition over all $2^{s}$ colors, $\frac{1}{\sqrt{2^{s}}} \sum_{c \in \mathcal{C}}|0\rangle|c\rangle$.

2. We then apply our exact quantum search algorithm recursively. For each color $c \in \mathcal{C}$ in quantum parallel, we search recursively among the vertices in $V_{c}$, hereby determining the root $v_{c} \in V_{c}$ of the subtree containing the leaf $\ell_{f(x)}$. Since $\left|V_{c}\right| \leq N^{\prime}$, this requires at most $F\left(N^{\prime}+1\right)$ queries to oracle $x$ and produces the superposition $\frac{1}{\sqrt{2^{s}}} \sum_{c \in \mathcal{C}}\left|v_{c}\right\rangle|c\rangle$. Since every vertex $v_{c}$ in this sum is on the path $\mathcal{P}$, we can rewrite the sum as

$$
\frac{1}{\sqrt{2^{s}}} \sum_{v \in \mathcal{P}}|v\rangle \sum_{c \in \mathcal{C}: v_{c}=v}|c\rangle
$$

3. We then apply the un-coloring operator $\mathrm{U}_{1}^{-1}$, producing the superposition $\frac{1}{\sqrt{2^{s}}} \sum_{v \in \mathcal{P}} \sqrt{p_{v}}|v\rangle|0\rangle$. Ignoring the second register which always holds a zero, this is

$$
\frac{1}{\sqrt{2^{s}}} \sum_{v \in \mathcal{P}} \sqrt{p_{v}}|v\rangle
$$

That is, we have (recursively) obtained a superposition over the vertices on the path $\mathcal{P}$ from the root of $\mathcal{T}$ to the parent of the leaf $\ell_{f(x)}$ labelled by $f(x)$.

4. We then apply the operator $\mathrm{U}_{2} \mathrm{O}_{x}^{\prime}$, producing the final state

$$
\mathrm{U}_{2} \mathrm{O}_{x}^{\prime} \frac{1}{\sqrt{2^{s}}} \sum_{v \in \mathcal{P}} \sqrt{p_{v}}|v\rangle=\frac{1}{\sqrt{2^{s}}} \sum_{v \in \mathcal{P}} \sqrt{p_{v}} \mathrm{U}_{2} \mathrm{O}_{x}^{\prime}|v\rangle
$$

which one can show equal to $\left|\ell_{f(x)}\right\rangle$. Thus, a final measurement of this state yields $f(x)$ with certainty.

The total number of queries to the oracle $x$ is at most $F\left(N^{\prime}+1\right)+1$, and thus, by Lemma 12, the algorithm uses at most $\log _{3}(N)+O(1)$ queries. Theorem 13 follows.

Theorem 13. The above described quantum algorithm for searching an ordered list of $N$ elements is exact and uses at most $\log _{3}(N)+O(1)$ queries. 


\section{Concluding remarks and open problems}

The inner product of two quantum states is a measure for their distinguishability. We have proposed a weighted all-pairs inner product argument as a tool for proving lower bounds in the quantum black box model. The possibility of using non-uniform weights seems particularly suitable when proving lower bounds for non-symmetric (possibly partial) functions. It could be interesting to consider other measures than inner products, as discussed, for instance, by Zalka [18], Jozsa and Schlienz [15], and Vedral [17].

The result of Grigoriev, Karpinski, Meyer auf der Heide, and Smolensky 13 implies that if only comparisons are allowed, the randomized decision tree complexity of element distinctness has the same $\Omega(N \log N)$ lower bound as sorting. Interestingly, their quantum complexities differ dramatically: the quantum algorithm by Buhrman et al. [8] uses only $O\left(N^{3 / 4} \log N\right)$ comparisons. There is still a big gap between this upper bound and our lower bound of $\Omega\left(N^{1 / 2} \log N\right)$. One way of closing this gab might be to consider quantum time-space tradeoffs, as has been done for the classical case [6. [.].

Our algorithm for searching an ordered list with complexity $\log _{3}(N)+O(1)$ is based on the classical binary search algorithm. The quantum algorithm initiates several independent walks/searches at the root of the binary search tree. These searches traverse down the tree faster than classically by cooperating, and they eventually all reach the leaf we are searching for in roughly $\log _{3}(N)$ steps. It could be interesting to consider if similar ideas can be used to speed up other classical algorithms. For instance one may consider other applications of operators like $\mathrm{U}_{2}$ acting on rooted trees and graphs.

\section{Acknowledgements}

We are grateful to Andris Ambainis, Harry Buhrman, Mark Ettinger, Gudmund S. Frandsen, Dieter van Melkebeek, Hein Röhrig, Daniel Wang, Ronald de Wolf, Andy Yao, and especially Sanjeev Arora, for their precious comments and suggestions.

\section{References}

1. Ambainis, A.: A better lower bound for quantum algorithms searching an ordered list. Proc. of 40th IEEE FOCS (1999) 352-357

2. Ambainis, A.: Quantum lower bounds by quantum arguments. Proc. of 32nd ACM STOC (2000) 636-643

3. Beals, R., Buhrman, H., Cleve, R., Mosca, M., de Wolf, R.: Quantum lower bounds by polynomials. Proc. of 39th IEEE FOCS (1998) 352-361

4. BeAme, P.: A general sequential time-space tradeoff for finding unique elements. SIAM J. Comput. 20 (1991) 270-277

5. Bennett, C.H., Bernstein, E., Brassard, G., Vazirani, U.: Strengths and weaknesses of quantum computation. SIAM J. Comput. 26 (1997) 1510-1523 
6. Borodin, A., Fischer, M. J., Kirkpatrick, D. G., Lynch, N A., Tompa, M.: A time-space tradeoff for sorting on nonoblivious machines. J. Comput. Sys. Sci. 22 (1981) 351-364

7. Brassard, G., Høyer, P., Mosca, M., TapP, A.: Quantum amplitude amplification and estimation. quant-ph/0005055, 2000

8. Buhrman, H., Dürr, C., Heiligman, M., Høyer, P., Magniez, F., Santha, M., DE Wolf, R.: Quantum algorithms for element distinctness. Proc. of 16th IEEE Computational Complexity (2001) (to appear)

9. Buhrman, H., DE Wolf, R.: A lower bound for quantum search of an ordered list. Inform. Proc. Lett. 70 (1999) 205-209

10. CHOI, M.-D.: Tricks or treats with the Hilbert matrix. Amer. Math. Monthly 90 (1983) 301-312

11. Farhi, E., Goldstone, J., Gutmann, S., Sipser, M.: A limit on the speed of quantum computation for insertion into an ordered list. quant-ph/9812057, 1998

12. Farhi, E., Goldstone, J., Gutmann, S., Sipser, M.: Invariant quantum algorithms for insertion into an ordered list. quant-ph/9901059, 1999

13. Grigoriev, D., Karpinski, M., Meyer auf der Heide, F., Smolensky, R.: A lower bound for randomized algebraic decision trees. Comput. Complexity 6 (1996/1997) 357-375

14. Grover, L. K.: Quantum mechanics helps in searching for a needle in a haystack. Phys. Rev. Letters 79 (1997) 325-328

15. Jozsa, R., Schlienz, J.: Distinguishability of states and von Neumann entropy. Phys. Rev. A 62 (2000) 012301

16. Shor, P. W.: Polynomial-time algorithms for prime factorization and discrete logarithms on a quantum computer. SIAM J. Comput. 26 (1997) 1484-1509

17. VedRAL, V.: The role of relative entropy in quantum information theory. quantph/0102094, 2001

18. ZalkA, Ch.: Grover's quantum searching algorithm is optimal. Phys. Rev. A 60 (1999) 2746-2751

Many of the above references can be found at the Los Alamos National Laboratory e-print archive (http://arXiv.org/archive/quant-ph). 